\title{
Relation microstructure-propriétés mécaniques d'un acier martensitique inoxydable
}

\author{
Guillaume Badinier $^{1}$, Jean-Denis Mithieux ${ }^{1}$ et Jean-Marc Herbelin ${ }^{2}$ \\ ${ }^{1}$ APERAM Research Center, BP. 15, 62330 Isbergues, France \\ ${ }^{2}$ APERAM Customer team Auto, BP. 15, 62330 Isbergues, France
}

\begin{abstract}
The relationship between microstructure and mechanical properties of MaX (1.4006) martensitic stainless steel has been studied. Optical microscopy was used to characterize the microstructure and the volume fraction of retained ferrite was measured by image analysis. Mechanical properties were measured in uni-axial tensile testing and a composite model has been developed to capture the effect of both the retained ferrite and the carbon content of the martensitic phase. First results show a reasonable correlation between the experimental stress-strain curves and the model. Results are discussed in view of a previous study on plain martensitic carbon steels.
\end{abstract}

\section{INTRODUCTION}

Les propriétés mécaniques des matériaux (métalliques entre autres) sont affectées par leur microstructure, et plus précisément par les dimensions caractéristiques de cette microstructure. Ainsi, dans les matériaux métalliques polycristallins usuels, c'est la taille de grain qui contrôle la limite d'élasticité, celle-ci étant d'autant plus élevée que la taille de grain est faible. Dans les matériaux métalliques plus complexes, comportant plusieurs phases par exemple, il est souvent observé que le comportement mécanique global est la moyenne des comportements mécaniques des différentes phases [1], ce qui implique de connaître précisément la microstructure (proportion de phase, dureté des phases, etc.) pour pouvoir interpréter le comportement mécanique correctement. Dans ce cas, c'est la phase la plus molle du matériau qui contrôle la limite d'élasticité globale et la plasticité est plus influencée par le contraste mécanique entre les phases que par la plasticité locale dans chacune des phases prises individuellement.

Nous proposons ici d'étudier un acier inoxydable martensitique bas carbone (0.1 \% pds), nommé MaX dans la suite de l'étude. Cette nuance est extrêmement intéressante car elle permet d'atteindre la cible $\mathrm{Rm}>1000 \mathrm{MPa}$ voulue par les constructeurs automobile pour des pièces de structure. Du fait de la grande dureté des aciers martensitiques, ces pièces de structure sont obtenues en emboutissage à chaud. Pour cela, les pièces sont montées en température (environ $950^{\circ} \mathrm{C}$, dans le domaine austénitique) dans un four classique, puis elles sont transférées dans une presse qui opère une double opération : l'emboutissage et le refroidissement simultané de la pièce jusqu'à la température ambiante. Les aciers inoxydables présentent un réel avantage par rapport aux aciers carbone à ce niveau puisqu'ils sont auto-trempants, c'est-à-dire qu'aucune transformation de phase ne vient gêner la transformation martensitique au refroidissement. Cependant, d'autres facteurs peuvent limiter leur utilisation industrielle, comme leur cinétique d'austénitisation (qui aura un impact sur les propriétés mécaniques du produit).
Tableau 1. Composition chimique du MaX (en \%pds).

\begin{tabular}{llllll}
\hline $\mathbf{C}$ & $\mathbf{M n}$ & $\mathbf{S i}$ & $\mathbf{C r}$ & $\mathbf{N b}$ & $\mathbf{N}$ \\
\hline 0,1 & 0,35 & 0,4 & 12,0 & 0,106 & 0,025
\end{tabular}

Les cinétiques d'austénitisation dans ces aciers sont connues pour être lentes [2], donnant en général un matériau comportant une phase ferritique douce et une phase martensitique très dure. Nous proposons ici une étude visant à comprendre l'influence d'une telle microstructure sur l'écrouissage à l'aide d'un modèle mécanique simple, appelé modèle de Masing [3].

\section{MÉTHODE EXPÉRIMENTALE}

La composition chimique du matériau étudié est présentée dans le tableau 1.

Les traitements thermiques ont été réalisés dans un four Nabertherm, et la trempe a été réalisée par air pulsé, ce qui donne une vitesse de refroidissement moyenne de $10^{\circ} \mathrm{C} / \mathrm{s}$. Le taux de ferrite résiduelle a été déterminé par analyse d'image, et le taux de carbone dans la phase martensitique a été estimé indirectement par la mesure de la température Ms. Les propriétés mécaniques des matériaux ainsi obtenus ont été mesurées en traction uniaxiale, à une vitesse de déformation de $10^{-3} \mathrm{~s}^{-1}$.

\section{MODÉLISATION DU COMPORTEMENT MÉCANIQUE}

Le comportement mécanique du MaX comportant de la ferrite et de la martensite est la moyenne du comportement mécanique des deux phases [4],

$$
\sigma_{m}=f_{\alpha} \sigma_{\alpha}+\left(1-f_{\alpha}\right)\left(\sigma_{0}+\bar{\sigma}\right) .
$$

Où $\sigma_{\alpha}$ est la contrainte dans la ferrite et $\bar{\sigma}$ la contrainte dans la martensite. L'évolution de la contrainte en fonction de la déformation dans la ferrite est décrite par une loi de Voce, 
Tableau 2. Caractéristiques de la microstructure.

\begin{tabular}{lclll}
\hline $\begin{array}{l}\text { Temp. } \\
\left({ }^{\circ} \mathrm{C}\right)\end{array}$ & $\begin{array}{c}\text { Temps } \\
(\mathrm{min})\end{array}$ & $\begin{array}{l}\mathrm{f} \alpha \\
(\%)\end{array}$ & $\begin{array}{l}\mathrm{Ms} \\
\left({ }^{\circ} \mathrm{C}\right)\end{array}$ & $\%$ pdsC \\
\hline 925 & 5 & 22 & 373 & 0,063 \\
925 & 60 & 7,5 & 373 & 0,063 \\
900 & 60 & 9,8 & 379 & 0,051 \\
950 & 15 & 10,1 & 361 & 0,086 \\
1025 & 5 & 10 & 354 & 0,1 \\
\hline
\end{tabular}

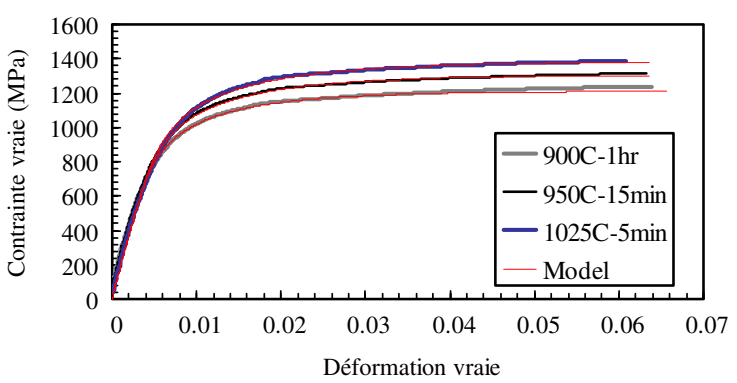

Figure 1. Modélisation des courbes contrainte-déformation du MaX austénitisé de façon à obtenir le même taux de ferrite résiduelle $(10 \%)$. Le modèle donne $\mathrm{m}=6,9, \mathrm{~m}=6,99$ et $\mathrm{m}=7,06$ après austénitisation a $900{ }^{\circ} \mathrm{C}$ pendant une heure, austénitisation à $950^{\circ} \mathrm{C}$ pendant 15 minutes et $1025^{\circ} \mathrm{C}$ pendant 5 minutes, respectivement. Tous les autres paramètres sont fixes.

et l'évolution de la contrainte de la martensite en fonction de la déformation est donnée par un modèle continu de Masing [3,5]. Ce modèle permet d'obtenir la contrainte moyenne d'un matériau «composite» contenant un spectre de contraintes. Dans ce cas, le spectre de contrainte est donné par la fonction statistique,

$$
f\left(\sigma_{l}\right)=\frac{1}{\sigma_{l} s \sqrt{2 \pi}} \exp \left(-\frac{\left(\ln \left(\sigma_{l}\right)-m\right)^{2}}{2 s^{2}}\right) .
$$

Où $\mathrm{m}$ et $\mathrm{s}$ sont des paramètres à déterminer, mais qui dépendent seulement du taux de carbone dans la martensite [6]. La contrainte moyenne est alors obtenue par l'intégration de l'équation,

$$
\frac{d \bar{\sigma}}{d \bar{\varepsilon}}=\frac{E\left(1-F\left(\sigma_{l}\right)\right)}{1+\frac{E}{\beta} F\left(\sigma_{l}\right)} .
$$

Où $\beta$ est un paramètre de localisation égal à $0,25 \mathrm{E}$ dans le cas présent [5].

\section{RÉSULTATS}

La microstructure présente les caractéristiques résumées dans le tableau 2.

Le modèle décrit plus haut a été testé sur quelques conditions et les résultats sont présentés sur les figures 1 et 2 .

La figure 1 montre qu'à taux de ferrite résiduelle équivalent, c'est la dureté de la martensite qui contrôle le comportement mécanique du produit. D'autre part, la

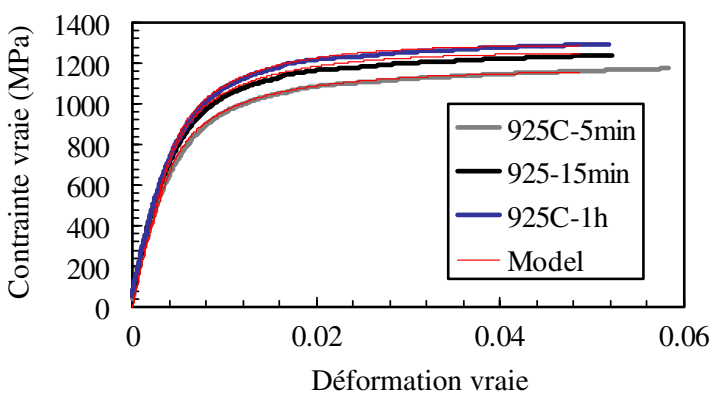

Figure 2. Modélisation des courbes contrainte-déformation du MaX austénitisé à $925^{\circ} \mathrm{C}$ pendant 5 (taux de ferrite de $22 \%$ ), 15 (taux de ferrite de $11,8 \%$ ) et 60 minutes (taux de ferrite de $7,5 \%$ ). Le modèle décrit plus haut est représenté en rouge, et a été obtenu en utilisant $\mathrm{m}=6,96$ et $\mathrm{s}=0,43$.

figure 2 montre que pour une température de recuit donné, seule le biphasage détermine les propriétés mécaniques du produit final.

\section{CONCLUSION}

La relation microstructure-propriétés mécaniques du MaX austénitisé de différentes manières (en variant le temps et la température) a été approfondie. Nous avons montré qu'un modèle simple permettait de décrire raisonnablement les courbes contrainte-déformation du produit, en connaissant le taux de ferrite résiduelle et le taux de carbone dans la martensite. Les résultats montrent que le paramètre $\mathrm{m}$ de l'équation (2) varie avec la composition en carbone nominale. Ces résultats semblent cohérents avec ceux obtenus sur des martensites au carbone plus faiblement alliées que les martensites d'aciers inoxydables étudiées ici.

\section{Références}

[1] Asaro, R. Elastic-plastic memory and kinematic-type hardening. Acta Met. 23, 1255-1265 (1975).

[2] Kostoj, V., Mithieux, J. D. \& Fröhlich, T. Influence of Chromium Carbide Size on the Austenitization Kinetics of a Martensitic Stainless Steel Measured by Dilatometry. Solid State Phenom. 172-174, 426-431 (2011).

[3] Masing, G. The Heyn theory of the stiffening of metals due to hidden elastic stresses. Wiss. Veroffentlichungen Aus Dem Siemens-Konzern 3, 231-239 (1923).

[4] Cho, K. \& Gurland, J. The law of mixtures applied to the plastic deformation of two- phase alloys of coarse microstructures. Met. Trans. 19, 2027-2040 (1988).

[5] Allain, S., Bouaziz, O. \& Takahashi, M. Toward a New Interpretation of the Mechanical Behaviour of As-quenched Low Alloyed Martensitic Steels. Isij Int. 52, 717-722 (2012).

[6] Badinier, G. Effect of carbon segregation and carbide precipitation on the mechanical response of martensite. (2013). 